\title{
Aplicabilidade clínica dos avanços da tecnologia CAD-CAM em Odontologia
}

\author{
Aloizio Filgueiras ${ }^{1}$ \\ Dione Gonçalves Pinto', \\ Lucas Lactim Ferrarez ${ }^{1}$, \\ Mariele Ferraz de Oliveira ${ }^{1}$, \\ Tamiris Alacoque de \\ Carvalho Freitas ${ }^{1}$ \\ - Bruno Salles Sotto-Maior ${ }^{2}$
}

${ }^{1}$ Programa de Pós-Graduação em Clínica Odontológica, Faculdade de Odontologia, Universidade Federal de Juiz de Fora. Juiz de Fora, MG

2Departamento de Odontologia Restauradora, Faculdade de Odontologia, Universidade Federal de Juiz de Fora. Juiz de Fora, MG $\triangle$ Bruno Sotto-Maior Rua Giuseppe Verdi, 910 Residencial São Lucas II CEP: 36036-643 Juiz de Fora - MG

乃 brunosottomaior@gmail.com

\section{RESUMO}

O avanço tecnológico tem se mostrado um grande aliado em diversas áreas, assim como na odontologia. O surgimento da tecnológica CAD/CAM (Computer Aided Design/Computer Aided Manufacturing) na década de 1950 trouxe outra realidade para a prática clínica diária. Previamente todo o processo laboratorial de trabalhos protéticos era demorado e puramente físico, atualmente a fabricação das peças protéticas demanda um menor tempo devido a utilização do meio digital, o que consequentemente diminui as consultas aos Cirurgiões-Dentistas. A adição de ferramentas digitais no planejamento e na execução dos tratamentos odontológicos alterou completamente o workflow, fazendo com que a busca para um aperfeiçoamento seja constante visando sempre o melhor atendimento e qualidade dos tratamentos realizados. Dessa forma este trabalho tem o objetivo de realizar uma revisão crítica da literatura sobre a aplicabilidade clínica da tecnologia CAD-CAM em Odontologia.

Palavras-chave: impressão tridimensional, tecnologia odontológica, CAD-CAM

\section{ABSTRACT}

The technological advance has been shown to be a greatly in several areas, as well as in dentistry. The emergence of the technological CAD / CAM (Computer Aided Design / Computer Aided Manufacturing) in the 1950 s brought another reality to daily clinical practice. Previously, the entire laboratory process of prosthetic work was time consuming and purely physical, currently the manufacture of prosthetic work requires less time due to the use of the digital medium, which consequently decreases the dentist appointments. The addition of digital tools in the planning and execution of dental treatments has completely altered the workflow, making the search for a constant improvement, always aiming at the best care and quality of treatment performed. Thus, this paper has the objective of performing a critical review of the literature on the clinical applicability of CAD / CAM technology in dentistry.

Keywords: 3D printing, dental technology, CAD-CAM 


\section{INTRODUÇÃO}

O progresso tecnológico que ocorre em diversos campos da ciência viabilizou aplicação também na área odontológica. Tecnologia aliada aos tratamentos odontológicos tem como objetivo a otimização dos procedimentos, busca por métodos de tratamento que conjugam estética, durabilidade, facilidade de execução e economia de tempo, tanto para o profissional quanto para o paciente, e isso já é uma realidade há mais de 30 anos na odontologia (ALGHAZZAWI, 2016). Os sistemas CAD/CAM (Computer Aided Design/Computer Aided Manufacturing), desenvolvido na década 1950, permitem modelar, projetar e fabricar objetos em processos industriais, mas tem sido utilizado na odontologia para a confecção de restaurações inlays, onlays, coroas, laminados, próteses parciais fixas e implantes (AHLHOLM et al., 2016), o que representa uma associação de sucesso entre a evolução da informática e da engenharia para as necessidades da odontologia.

Os maiores desenvolvimentos quanto à tecnologia CAD/CAM na odontologia se iniciaram nas décadas de 70 e 80 pelos pesquisadores François Duret, Werner Moermann e Matts Andersson, idealizadores dos sistemas Sopha ${ }^{\circledR}$ System, CEREC $\AA$ e Procera ${ }^{\circledR}$ respectivamente (MORMANN, 2006; CORREIA 2006). O sistema Sopha ${ }^{\circ}$ System foi pioneiro na produção de restaurações protética com morfologia oclusal, mas não obteve sucesso devido à sua alta complexidade e custo (DAVIDOWITZ; KOTICK, 2011). Atualmente, várias empresas têm desenvolvido tais sistemas com alta tecnologia, que se baseiam em três componentes fundamentais: sistema de leitura do preparo dental, podendo ser intraoral ou laboratorial (escaneamento), software de desenho da peça protética (CAD) e sistema de fresagem (CAM) (ALGHAZZAWI, 2016; JODA, 2017).

Ao início da utilização da tecnologia CAD/CAM os sistemas cerâmicos utilizados inicialmente foram as cerâmicas feldspática, leucita e dissilicato de lítio, os blocos em compósito também eram utilizados (DAVIDOWITZ; KOTICK, 2011). Nos anos 2000, o sistema CEREC $\otimes 1$ CEramic REConstruction, deu espaço para a terceira geração, CEREC $® 3$, este sistema apresentou avanços em relação à adaptação das restaurações, melhor obtenção da anatomia oclusal e maior rapidez no desgaste do bloco cerâmico (SANNINO et. al., 2015). Incorporou-se também, a tridimensionalidade nos seus softwares integrado a um computador. A unidade escaneadora foi aperfeiçoada utilizando o método do princípio da triangulação ativa, com a captura óptica da imagem realizada por infravermelho, produzindo um sinal elétrico e gerando dados em três dimensões na tela do computador (DAVIDOWITZ; KOTICK, 2011). Dessa forma o escâner projeta um padrão linear sob um ângulo de triangulação ativa, permitindo que as linhas luminosas não apareçam planas, mas com deslocamentos suaves, dependendo da profundidade do preparo. A unidade fresadora foi separada da digitalização e apresenta duas pontas montada diamantadas, uma cilíndrica e outra tronco-cônica, melhorando consideravelmente as anatomias das peças restauradoras (MORMANN, 2006).

Entre 2001 e 2003, ocorreu a criação do CEREC inLab, um sistema de laboratório que permitia a digitalização à laser do modelo de gesso, onde a infraestrutura é desenhada no computador (CAD) e, posteriormente, executada a usinagem do bloco de cerâmica e posteriormente seria aplicada a cerâmica de cobertura (MORMANN, 2006). Já o CEREC 3D trouxe uma tecnologia muito mais ilustrativa, com manipulação do sistema de forma mais fácil e intuitiva, permitindo que as restaurações pudessem ser avaliadas na tela, de todos os ângulos (LIU, 2005; MORMANN, 2006; MANTRI; BHASIN, 2010).

O sistema Procera System $\AA$ foi desenvolvido pelo Dr. Matts Andersson no início da década de 80, buscando fazer o processamento do titânio devido problemas técnicos durante sua fundição (ANDERSON, 1996; MIYASAKI, 2011) e conseguinte, em 1989, foi desenvolvido para o Procera Allceram $\AA$, no qual estruturas eram produzidas com alta precisão com o uso de alumina densamente sinterizada (MORMANN, 2006). Atualmente, a empresa disponibiliza um sistema CAD/CAM completo, somente para fluxo de trabalho laboratorial, o 3Shape Dental System $\AA$, em que se tem o escaneamento intraoral com o 3Shape TRIOS $\AA$ e o escaneamento de bancada o 3Shape da série E e 3Shape D1000® e D2000®.

Como Duret afirmou em seu artigo no ano 1991, concluindo que o CAD/CAM traria efeitos importantes na odontologia, aumentaria a versatilidade, a precisão, a relação custo-benefício e faria parte da prática odontológica de rotina no início do século XXI (DURET; PRESTON, 1991), se confirmam atualmente. Sendo assim, o objetivo desta revisão é apresentar o panorama atual da aplicabilidade da tecnologia CAD/CAM na confecção de próteses fixas em Odontologia.

\section{REVISÃO DE LITERATURA}

Para a realização desta revisão, foi realizada uma busca em bases de dados, como, Pubmed e Scielo. Onde as palavras-chave utilizadas foram CAD/CAM, CEREC system, Procera system, digital impressions, conventional impressions

Será exposto a seguir as etapas do workflow digital.

\section{Escaneamento}

A primeira etapa no processo de confecção de um trabalho em CAD/CAM é o escaneamento, no qual se tem a obtenção de estruturas tridimensionais. Podendo este ser feito de duas maneiras: intraoral e/ou escaneamento 
de bancada (ALGHAZZAWI, 2016).

Com o escâner intraoral a imagem tridimensional é obtida diretamente do meio bucal, eliminando as etapas convencionais de moldagem, consequentemente eliminando possíveis distorções dos materiais de moldagem, garantindo maior confiabilidade e ganho em tempo nas etapas clínicas (BIRNBAUM; AARONSON, 2008; KHALED; AHMED, 2018). Mas o sistema também apresenta algumas desvantagens como a necessidade de obtenção de um escâner no consultório. Em determinadas situações que o escâner deveria atuar, como em sulcos gengivais ou preparos mais profundos, o profissional encontrará uma maior dificuldade na obtenção destas imagens (RAU et. al., 2017).

No escâner de bancada, a imagem tridimensional é obtida fora do meio bucal, podendo ser feita a partir do molde ou do modelo de gesso, o que seria vantajoso em relação ao escâner intraoral, pois a fidelidade de cópia é maior (HACK; PATZELT, 2015).

\section{Software}

Um software específico é fornecido pelo fabricante, sendo que cada marca comercial possui um software distinto. Tais softwares possuem bibliotecas digitais, dais quais se pode obter coroas anatômicas completas, inlays, onlays, table tops, facetas, pilares personalizados, guia cirúrgicos, dentre outros. Tais peças ainda podem ser personalizadas de acordo com cada paciente, podendo ser alterado sua forma e tamanho (ALGHAZZAWI, 2016).

\section{Imagens por Ressonância Magnética para avaliação de Processos Patológicos}

Diversas lesões podem acometer o sistema estomatognático, como os cistos e tumores e as técnicas de diagnóstico por imagem são muito importantes para complementar e auxiliar o diagnóstico correto (PINTO et al., 2016). A IRM é a modalidade de escolha para o diagnóstico de tumores, pois exibe um bom contraste entre tecidos normais e anormais, além de possibilitar a identificação do conteúdo da lesão, a vascularização local e o possível acometimento da cadeia ganglionar (KABALA et al., 1992). Ademais, para os tumores malignos de cabeça e pescoço, a informação sobre o estadiamento e diagnóstico de recidivas, são fundamentais para o planejamento do tratamento, para um melhor prognóstico e menor morbidade para o paciente (BONDT et al., 2007; SARRIÓN et al., 2015).

Juerchott et al. (2018) estabeleceram, a partir de IRM, critérios para a diferenciação de cistos periapicais e granulomas in vivo, tais como a análise das margens da lesão (cistos: bem definidos; granulomas: mal definidos), textura da borda (cistos: borda homogênea; granulomas: borda heterogênea), textura do centro da lesão, envolvimento do tecido circundante (cistos: não envolvem; granulomas: envolvem), espessura máxima da margem da lesão, dentre outros. Do total, seis critérios foram capazes de diferenciar adequadamente as lesões após comparação com o resultado histopatológico, fornecendo um impacto clínico relevante, pois o tratamento é específico para cada condição. No entanto, com a IRM, não foi possível determinar o tipo de cisto.

\section{Processamento}

A obtenção de peças protética por meio da tecnologia CAD pode se dar por dois tipos de processamento, o subtrativo, fresagem, e o aditivo, impressão 3D (ALGHAZZAWI, 2016).

\section{Fresagem}

A fresagem corresponde à última fase do sistema CAD/CAM, que seria a confecção da peça protética propriamente dita. Para tal, existem duas formas de obtenção, através da produção chairside, laboratorial e outsourcing (ALGHAZZAWI, 2016).

A produção chairside, é realizada no próprio consultório pelo dentista, ou seja, a restauração indireta é produzida e cimentada em sessão única. Os tipos de restaurações confeccionadas desta forma são; inlays, onlays, overlays, coroas (sem copings) e facetas(HILGERT et al., 2010). Ela apresenta como desvantagem $O$ fato dessas restaurações serem usinadas a partir de blocos de materiais restauradores pré-fabricados, nos quais a cor é única ou com variação de saturação e translucidez padronizada, prejudicando a estética em dentes anteriores; além disso, as cerâmicas geralmente não são glazeadas, e sim polidas (HILGERT et al., 2009; HILGERT et al., 2009; HILGERT et al., 2010).

Na produção laboratorial, as peças protéticas são fresadas em laboratórios. Inicialmente a digitalização do preparo para a obtenção do modelo virtual é realizada, e a mesma pode ser de duas formas: impressão óptica, com um escâner intraoral, ou impressão convencional, modelagem em gesso e digitalização, com escâner extraoral. Em ambos os casos, o dentista envia os dados ou impressão/modelagem para o laboratório de prótese que consegue produzir os mais variados tipos de peças protéticas, desde inlays, onlays e coroas unitárias, até infraestruturas de 14 elementos, abutments personalizados para implantes, restaurações provisórias em acrílico, infraestruturas para próteses removíveis, etc (ALGHAZZAWI, 2016). A vantagem deste tipo de produção se dá pela possibilidade de caracterização extrínseca e glazeamento nos materiais cerâmicos (HILGERT et al., 2009; HILGERT et al., 2009; HILGERT et al., 2010).

o tipo de produção outsourcing é realizado em centros de produção, afiliados às empresas fornecedoras do sistema CAD/CAM, como, por exemplo: LAVA (3M ESPE $®$ ), ETKON (Straumann $®$ ) e PROCERA (Nobel Biocare $\AA$ ). Nesta possibilidade, tanto dentistas 
quanto laboratórios, que optam por não adquirir os sistemas CAD/CAM, enviam seus trabalhos para estes locais altamente estruturados. Esses centros possuem equipamentos mais complexos e tecnologicamente avançados, com os quais é possível produzir diversos tipos de estruturas (HILGERT et al., 2009; HILGERT et al., 2009; HILGERT et al., 2010).

\section{Impressão 3D}

A impressão 3D, atualmente, faz uso de duas técnicas, o processamento digital de luz e a impressão polyjet. A impressão por processamento digital de luz é feita utilizando uma fonte de luz convencional, painel de cristal líquido, ou fonte de projeção para polimerizar a camada superficial de uma cuba de resina fotopolimerizável em um formato pré-definido pelo modelo digital. A impressão polyjet é realizada com cabeças de jato que borrifam ou injetam a resina nas áreas desejadas. À medida que as cabeças do jato fazem passagens subsequentes, cada camada pulverizada é polimerizada com uma fonte de luz ultravioleta (BROWN et al., 2018).

\section{DISCUSSÃO}

A odontologia digital está cada vez mais presente nos consultórios e laboratórios de próteses dentárias e como consequência grande parte dos tratamentos reabilitadores podem ou são planejados e executados do seu início ao fim de maneira digital. Tecnologias como a radiografia digital, tomografia cone bean, câmeras intraorais, fotografia digital, microscópio e escâneres de boca auxiliam no planejamento e os equipamentos como, fresadoras, impressores 3D, escâneres de bancada guiaram o trabalho técnico laboratorial (BIRNBAUM, 2010; POLIDO, 2010; AHMED, 2018; YILMAZ, 2015).

Para se obter êxito nos tratamentos conduzidos com o auxílio da odontologia digital tem-se em mente que existem dois campos distintos, o mundo digital e o mundo físico. Os pacientes (físico) precisam ser digitalizados (digital) para se realizar o tratamento. No futuro as atuações em boca serão cada vez menores e os modelos digitais dos pacientes fornecerão informações suficientes para o planejamento e a execução do tratamento. É possível, até mesmo, pensar que no futuro a mão de obra direta do Cirurgião-Dentista possa ser substituída por um robô, visando obter uma maior precisão. O Cirurgião-Dentista irá se especializar e se qualificar para o manuseio de equipamentos e diagnosticar problemas orais "deixando de lado" a atividade prática odontológica (BIRNBAUM, 2010; POLIDO, 2010; ALGHAZZAWI, 2016; TAKEUCHI et al., 2018; AHMED, 2018) Sendo assim um protocolo de trabalho clínico digital deverá ser criado para execução dos procedimentos, como por exemplo, captura e processamento de imagens digitais: câmeras fotográficas digitais, intraorais e filmadoras, escâneres intraorais ou de bancada e exames de imagem como tomografia e radiografa digital serão utilizadas para ser obter a versão digital do paciente. (BIRNBAUM, 2010; ALGHAZZAWI, 2016; TAKEUCHI et al., 2018; AHMED, 2018). Realização de um planejamento virtual, após a captura das informações, estas serão analisadas através de softwares e todo tratamento será conduzido com essas informações desde o planejamento até a execução. Desta forma o profissional deverá gastar mais tempos na frente de equipamentos digitais do que na frente do próprio paciente. (BIRNBAUM, 2010; ALGHAZZAWI, 2016; TAKEUCHI et al., 2018; AHMED, 2018).

Feito o planejamento e a última etapa será o processo de confecção por fresagem ou impressão 3D, a partir do planejamento virtual a manufatura será executada retornando assim ao mundo físico, transformando o planejamento digital em próteses dentais fixas, removíveis, parciais e totais, guias cirúrgicos, alinhadores dentais, placas de bruxismo, dentre outros. Através de duas técnicas básicas a fresagem (forma subtrativa) ou a impressão 3D (forma aditiva) são criados os trabalhos que serão destinados à reabilitação dos pacientes.

As câmeras intraorais comercialmente disponíveis se apresentam de duas maneiras: câmeras de imagem única que registram imagens individuais, sendo elas, iTero (Align Technology), o PlanScan (Planmeca), o CS 3500 (Carestream Dental LLC) e o Trios (3 Shape). E as câmeras de vídeo, que são usadas pelo scanner True Definition (Lava Chairside Oral Scanner), Apollo DI (Sirona) e Sistemas OmniCam system (Sirona). As câmeras de imagem única registram apenas uma secção, sendo necessária a sobreposição de imagens pelo software para a geração de um modelo de arcada completa tridimensional (ALGHAZZAWI, 2016).

Dentre os sistemas CAD/CAM disponíveis para consultórios odontológicos temos o CEREC e E4D, e uma das vantagens do sistema E4D em relação ao CEREC é a eliminação de um dos passos, no caso a aplicação de uma camada de dióxido de titânio. Já para os sistemas laboratoriais completos temos, Sirona CEREC inLab, Degudent Cercon, Kavo Everest e Wieland Zeno (HILGERT et al., 2009). Cada sistema possui sua peculiaridade, seja em relação as etapas necessárias para finalização das restaurações, ao tipo de material disponível e ao grau de dificuldade para sua utilização.

Com a utilização de tal tecnologia obtêm-se tratamentos protéticos cada vez mais bem adaptados e com um tempo muito menor de tratamento, mas os usos de peças protéticas confeccionadas de forma tradicional, através das técnicas de moldagem, ainda apresentam boa adaptação marginal e longevidade (BIRNBAUM, 2010; YILMAZ, 2015; ALGHAZZAWI, 2016; TAKEUCHI et al., 2018; AHLHOLM et al., 2018; AHMED, 2018). Estudos indicam que coroas totais confeccionadas pelo CAD/CAM apresentam uma desadaptação de $49 \mu \mathrm{m}$, já as coroas produzidas através da moldagem tal nível de desadaptação é de $71 \mu \mathrm{m}$, porém aceitável clinicamente (SYREK et al., 2010; TING-SHU, JIAN, 2015).

Apesar da tecnologia a serviço da odontologia ainda 
possuir um custo muito elevado, o que inviabiliza a aquisição de equipamentos, esta vem diminuindo seus valores de mercado e em um futuro próximo será possível que todo Cirurgião-Dentista implemente um fluxo de trabalho digital em seu consultório, clínica ou laboratório (ALGHAZZAWI, 2016; AHMED, 2018). Equipamentos cada vez menores mais modernos e compactos ajudam a otimizar o espaço dentro do ambiente de trabalho e profissionais que possuem mais de um local de trabalho poderão através de redes fechadas ou abertas de computadores trabalharem com comunicação remota utilizando sua estrutura digital a distância (POLIDO, 2010; ALGHAZZAWI, 2016; AHMED, 2018)

Com isso temos a certeza que as reabilitações orais ganharam destaque nos tempos modernos e nos mostram novas perspectivas para o futuro (BIRNBAUM, 2010; POLIDO, 2010; TING-SHU; JIAN, 2015TAKEUCHI et al., 2018; AHMED, 2018).

\section{CONCLUSÃO}

Com os constantes avanços, fica evidente que o uso dessas tecnologias já é uma realidade no dia a dia clínico dos profissionais em diferentes áreas da odontologia. O uso da tecnologia CAD/CAM para confecção de próteses fixas tem se mostrado vantajoso tanto por questões financeiras, quanto pela praticidade e agilidade de tratamento; possibilitando os cirurgiões-dentistas a realizarem tratamentos mais eficazes, com maior precisão e menor tempo. Portanto, cabe a nós definirmos o quão importante é a nossa inserção neste workflow digital, analisando nossa área de atuação e o mercado ao qual estamos inseridos. Desta maneira, independente da escolha do fluxo de trabalho, analógico ou digital, estaremos conscientes da busca constante pelo melhor atendimento.

\section{REFERÊNCIAS}

ANDERSSON, M. et al. Accuracy of machine milling and spark erosion with a CAD/CAM system. Journal of Prosthetic Dentistry, v. 76, n. 2, p. 187-193, Aug. 1996.

ANDERSSON, M. et al. Procera: a new way to achieve an all-ceramic crown. Quintessence International., v. 29, n. 5, p. 285-296, May. 1998.

AHLHOLM, P. et al. Digital versus conventional impressions in fixed prosthodontics: A review. Journal of Prosthodont, v. 27, n. 1, p. 35-41, Jan. 2018.

AHMED, K.E. We're going digital - the current state of CAD/CAM in prosthodontics. Primary Dental Journal, v. 7, n. 2, p. 30-35, Summer. 2018.

ALGHAZZAWI, T.F. Advancements in CAD/CAM technology: Options for practical implementation. Journal of Prosthodontic Research, v. 60, n. 2, p. 72-84, Apr. 2016.
BIRNBAUM, N.S. The revolution in dental impressioning. Inside Dentistry, v. 6, n. 7, p.1-3, 2010.

BIRNBAUM, N.S., AARONSON, H.B. Dental impressions using 3D digital scanners: virtual becomes reality. Compendium of Continuing Education in Dentistry, v. 29, n. 8, p. 494-505, Oct. 2008.

BROWN, G.B et al. Accuracy of 3-dimensional printed dental models reconstructed from digital intraoral impressions. American Journal of Orthodontics and Dentofacial Orthopedics, v. 154, n.5, p. 733-739, Nov. 2018.

DAVIDOWITZ, G., KOTCK, P.G. The use of CAD/CAM in dentistry. Dental Clinics of North America, v. 55, n. 3, p. 559-570, Jul. 2011.

HACK, G.D., PATZELT, S.B. Evaluation of the accuracy of six intraoral scanning devices: an in-vitro investigation. ADA Professional Product Review, v. 10, n. 4, p. 1-5, Set. 2015.

HILGERT, L.A. et al. Odontologia restauradora com sistemas CAD/ CAM: o estado atual da arte. Parte 1: princípios de utilização. Clínica - international journal of brazilian dentistry, v. 5, n. 3, p. 294-303, Set. 2009.

HILGERT, L.A. et al. Odontologia restauradora com sistemas CAD/ CAM: o estado atual da arte. Parte 2: possibilidades restauradoras $e$ sistemas CAD/CAM. Clínica- International Journal of Brazilian Dentistry, v. 5, n. 4, p. 424-435, Dec. 2009.

HILGERT, L.A. et al. Odontologia restauradora com sistemas CAD/ CAM: o estado atual da arte. Parte 3: materiais restauradores para sistemas CAD/CAM. Clínica- International Journal of Brazilian Dentistry, v. 6, n. 1, p. 86-96, Mar. 2010.

JODA, T., ZARONE, F., FERRARI, M. The complete digital workflow in fixed prosthodontics: a systematic review. BMC Oral Health, $v$. 17, n. 124, p. 3-9, Sep. 2017.

LIU, P.R. A panorama of dental CAD/CAM restorative system. Compendium of Continuing Education in Dentistry, v. 26, $n$. 7, p. 507-512, Jul. 2005.

MIYAZAKI, T., HOTTA, Y. CAD/CAM systems available for the fabrication of crown and bridge restorations. Australian Dental Journal, v. 56, n. 1, p.97-106, Jun. 2011.

MORMANN, H.W. The evolution of the CEREC system. The Journal of the American Dental Association, v. 137, p. 7s-13s, Sep. 2006.

POLIDO, W.D. Moldagens digitais e manuseio de modelos digitais: o futuro da Odontologia. Dental Press Journal of Orthodontics, v. 15, n. 5, p. 18-22, Sep. 2010. 
RAU, C.T. et al. The quality of fixed prosthodontic impressions: an assessment of crown and bridge impressions received at commercial laboratories. The Journal of American Dental Association, v. 148, n. 9, p. 654-660, Sep. 2017.

SANNINO, G. et al. Cerec CAD/CAM chairside system. Oral Implantology, v. 7, n. 3, p. 57-70, Apr. 2015.

SYREK, A. et al. Clinical evaluation of all-ceramic crowns fabricated from intraoral digital impressions based on the principle of active wavefront sampling. Journal of dentistry, v. 38, n. 7, p. 553-559, Jul. 2010.

TAKEUCHI, Y. et al. Use of digital impression systems with intraoral scanners for fabricating restorations and fixed dental prostheses. Journal of Oral Science, v. 60, n. 1, p. 1-7, Dec. 2018.

TING-SHU, S., JIAN, S. Intraoral digital impression technique: a review. Journal of Prothodontics, v. 24, n. 4, p. 313-321, Jun. 2015.

YILMAZ, B. Incorporating digital scans of diagnostic casts into computed tomography for virtual implant treatment planning. The Journal of Prosthetic Dentistry, v. 114, n. 2, p. 178-181, Aug. 2015. 